\title{
Initiation of Grain Growth Observed Using Electrostatic-Subframing
}

B.W. Reed. ${ }^{1}$, A.M. Monterrosa ${ }^{2}$, A.A. Moghadam ${ }^{1}$, R.S. Bloom ${ }^{1}$, S.T. Park ${ }^{1}$, S.A. Briggs ${ }^{2,3}$, P.M. Price ${ }^{2}$, C.M. Barr², J.T. McKeown' ${ }^{4}$ D.J. Masiel ${ }^{1}$, K. Hattar²*

1. Integrated Dynamic Electron Solutions, Inc., Pleasanton, CA 94588, USA

2. Sandia National Laboratories, Albuquerque, NM 87123, USA

3. Oregon State University, Corvallis, OR 97331, USA

4. Lawrence Livermore National Laboratory, Livermore, CA 94551, USA

* Corresponding author: khattar@sandia.gov

While in-situ TEM offers an avenue to study dynamic nanoscale processes, it often suffers from poor temporal resolution. The limiting factor for time resolution in many conventional TEM systems is usually the camera. Advances in ultrafast in-situ microscopy and dynamic TEM (DTEM) have attempted to solve this issue by creating nanosecond or faster electron pulses to capture snapshots as events occur $[1,2]$. However, these designs are often limited to single snapshots and require extensive modifications to the TEM system. The Relativity electrostatic subframing system [3] allows for the acquisition of in-situ videos of events occurring at the multi-kHz time scale. The multi-MHz deflector system deflects a conventional electron beam onto multiple areas of the TEM camera, effectively dividing a single exposure into many frames. Up to 16 frames can be created from a single camera exposure, and with the help of compressive sensing, 100+ frames of video are possible.

The I ${ }^{3}$ TEM facility at Sandia National Laboratories [4] was modified with the Relativity deflector system and with the addition of an infrared sample drive laser. The laser is a 20W, 1064 nm wavelength IR beam and is pulsed at a frequency of $33 \mathrm{kHz}$ with a $36 \mathrm{~ns}$ pulse width aligned to the sample location. This laser is used to initiate reactions, phase transformations, or grain growth in the target sample which is then subsequently imaged by the Relativity deflector system.

In this study, a film of 50 nm-thick nanocrystalline gold was exposed to a 36 ns IR pulse to initiate grain coarsening. The grain evolution over approximately $52 \mathrm{~ms}$ was captured using the Relativity deflector system. Figure 1 shows the grain coarsening captured using 1-to-1 deflector mode, resulting in 16 frames over the course of the event. The first frame is sacrificial during which the beam blanker on the TEM is stabilizing. The second frame shows the nanocrystalline microstructure before laser exposure. The laser is turned on during the third frame and the subsequent grain coarsening occurs in the following frames. Coarsening of the grains occurs very quickly, with their size increasing to near the final size by the fourth frame. Finer time resolution enabled with compressive sensing mode revealed that grain growth had nearly completed by $400 \mu$ s after laser initiation. This observation agrees well with theory of grain growth in thin films by Thompson [5], which predicts an initial stage of rapid grain growth, followed by an arrest in grain growth as the grains reach sizes of 2-3x the film thickness. The findings in this study highlight the power of high temporal resolution at the nanoscale and the potential for discovering the previously unknown mechanisms of grain growth. ${ }^{6}$ 
References:

[1] N.D. Browning, et al., Curr. Opin. Solid State Mater. Sci. 16, (2012), p. 23-30.

[2] T. LaGrange, B.W. Reed, and D.J. Masiel, MRS Bull. 40, (2015), p. 22-28

[3] B.W. Reed, et al., Microsc. Microanal. 23 (Suppl 1), (2017), p. 84

[4] K. Hattar, D.C. Bufford, and D.L. Buller, Nucl. Instrum. Methods Phys. Res. Sect. B Beam Interact. Mater. At. 338, (2014) p. 56-65.

[5] C.V. Thompson, Annu Rev. Mater. Sci. 20, (1990) p. 245-268.

[6] This work was supported by the US Department of Energy, Office of Basic Energy Sciences. Sandia National Laboratories is a multi-mission laboratory managed and operated by National Technology and Engineering Solutions of Sandia, LLC., a wholly owned subsidiary of Honeywell International, Inc., for the U.S. Department of Energy's National Nuclear Security Administration under contract DE-NA0003525. This material is based in part upon work supported by the U.S. Department of Energy, Office of Science, Office of Basic Energy Sciences, under Award Number DE-SC0013104.

SAND2019-1650 C

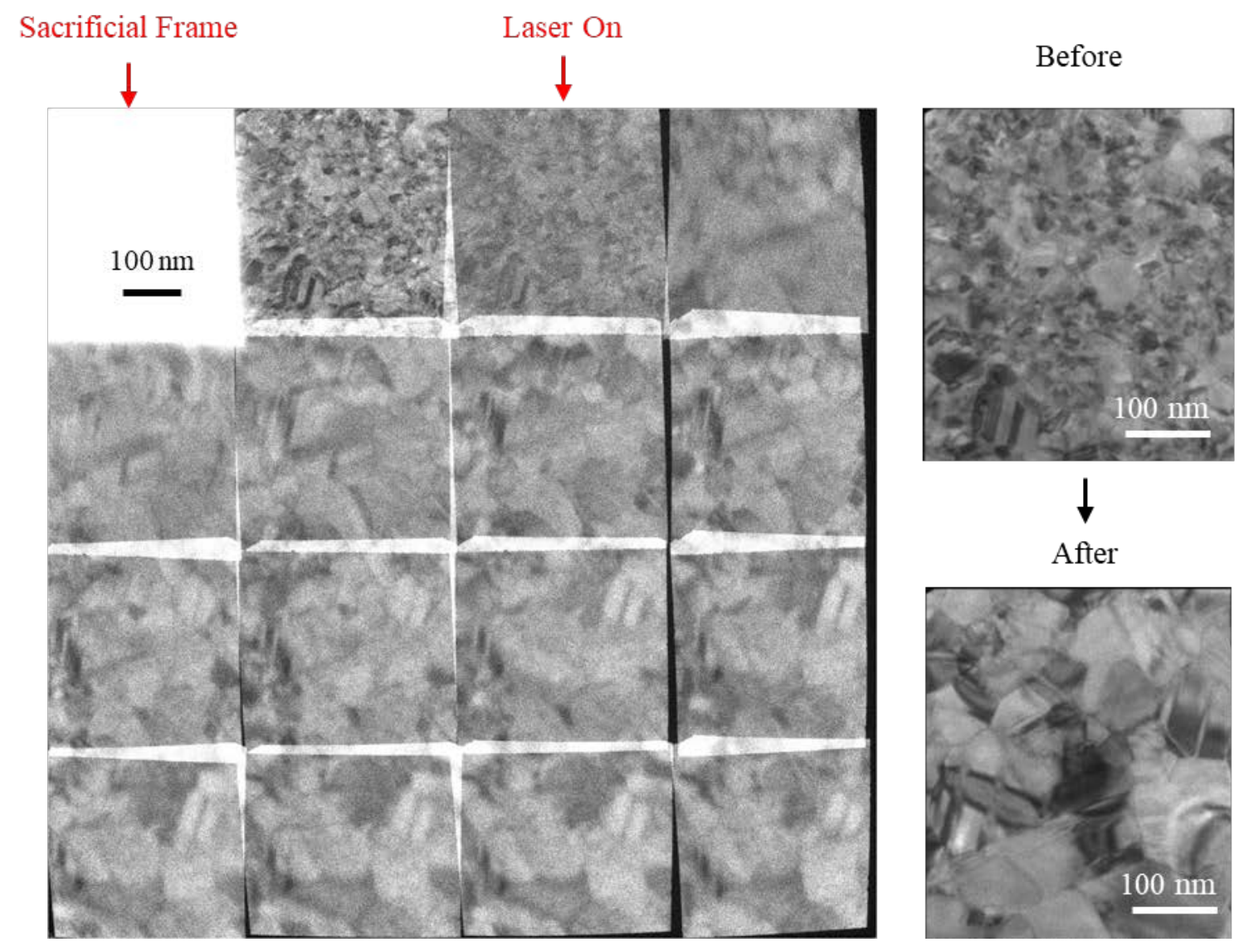

Figure 1. The grain coarsening evolution of nanocrystalline gold is captured during an IR laser shot in 16 frames using the Relativity deflector system in 1-to-1 mode. The initial and final microstructures are shown on the right. The first frame is a saturated sacrificial frame during which the beam blanker was stabilizing. The laser pulse was initiated during the third frame. 